\title{
Analysis of potential key genes in very early hepatocellular carcinoma
}

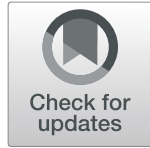

Min $\mathrm{Wu}^{1 \dagger}$, Zhaobo Liu' ${ }^{1 \dagger}$, Xin $\mathrm{Li}^{1}$, Aiying Zhang ${ }^{2}$, Dongdong Lin ${ }^{1}$ and Ning $\mathrm{Li}^{1^{*}}$

\begin{abstract}
Background: Hepatocellular carcinoma (HCC) is the major pathological type of primary liver cancer, one of the leading causes of cancer death worldwide. In addition, the long-term survival rates of HCC still remain low. Therefore, we attempted to identify the potential key genes in the occurrence of HCC by comparing the expression profiles of very early HCC tissue samples with that of chronic cirrhotic tissue samples by integrating the bioinformatics analysis in this study.

Methods: Gene expression profiles of 19 very early HCC and 19 cirrhotic tissue samples were selected from GSE63898. Differentially expressed genes (DEGs) were also identified by using online tool GEO2R. Furthermore, the GO and KEGG enrichment analysis of the DGEs were conducted on DAVID datasets. Then a protein-protein interaction (PPI) network was constructed and the modules were analyzed based on STRING database and Cytoscape software. The hub genes were screened by applying the cytoHubba plugin and then analyzed with the Kaplan Meier plotter.
\end{abstract}

Results: A total of 118 DEGs were identified between very early HCC and cirrhotic tissue samples. These DGEs were strongly associated with several biological processes, such as negative regulation of growth and p53 signaling pathway. A PPI network was constructed and top eight hub genes, including CDKN3, CDK1, CCNB1, TOP2A, CCNA2, CCNB2, PRC1, and RRM2, were determined. High expressions of CDK1, CCNB1, TOP2A, CCNA2, PRC1, RRM2, CDKN3, and CCNB2 were associated with poorer overall survivals (OS) in HCC patients.

Conclusion: We had compared the expression profiles between the very early HCC and cirrhotic tissue samples by using bioinformatics analysis tools, which might help us better to understand the molecular mechanism of the initiation of HCC and even to find novel targets for HCC therapy.

Keywords: Bioinformatics analysis, Protein-protein interaction network, Differentially expressed genes, Hepatocellular carcinoma

\section{Introduction}

Primary liver cancer remains one of the leading causes of cancer death and is a major public health problem all over the world. It was reported that the incidence rate of primary liver cancer continued to increase, and there were 42,220 estimated new primary liver cancer cases yearly in the USA [1]. However, the incidence rate of liver cancer was predicted to decrease in most Asian countries, except for Thailand [2]. Hepatocellular carcinoma $(\mathrm{HCC})$ is the major pathological type of primary

\footnotetext{
* Correspondence: liningyouan@163.com

${ }^{\dagger}$ Min Wu and Zhaobo Liu contributed equally to this work.

'Department of General Surgery, Beijing Youan Hospital, Capital Medical

University, 8 Xitoutiao, Youanmenwai, Feng-tai District, Beijing 100069, China Full list of author information is available at the end of the article
}

liver cancer. The mortality and incidence rates of HCC remain high in Southeast Asia and Africa, where the infection of hepatitis B virus is endemic [3, 4]. Most cases of HCC in these areas are secondary to chronic liver cirrhosis. In contrast, the HCC incidence rate is relatively low in Europe, Australia, and North America, where the major etiologies are chronic alcoholism, iron storage disorder and exposure to aflatoxin [5].

Although the remarkable improvements are achieved in the treatment of HCC such as liver transplantation, radical surgical resection, and interventional therapy [6-8], the long-term survival rates of HCC still remain low worldwide. One of the major reasons is that most patients with HCC were diagnosed at advanced stages. It is crucial to

(c) The Author(s). 2019 Open Access This article is distributed under the terms of the Creative Commons Attribution 4.0 International License (http://creativecommons.org/licenses/by/4.0/), which permits unrestricted use, distribution, and 
find out new therapeutic targets and novel diagnostic biomarkers for the early diagnosis and timely treatment of HCC. Therefore, it is still urgent to further explore the exact molecular mechanisms of the development, progression, invasion, and metastasis of HCC.

An increasing number of studies have revealed that the initiation of $\mathrm{HCC}$ was a complicated process that associated with multiple cellular pathways and numerous genes alterations [9-11]. In addition, Gene Expression Omnibus (GEO) is a public functional genomics data repository that offers us an opportunity to mine the gene expression profiles of all kinds of cancers. Therefore, in this study, we tried to identify and evaluate the clinical values of the potential key genes in the occurrence of HCC by comparing the expression profiles of very early HCC tissue with that of chronic cirrhotic tissue samples by integrating the bioinformatics analysis.

\section{Material and methods}

Gene expression data of HCC and cirrhotic tissue samples Gene chip data of 19 very early HCC and 19 cirrhotic tissue samples, which were selected from GSE63898 gene expression profile, was downloaded from GEO. The dataset was provided by Augusto Villanueva, et al and performed on the Affymetrix Human Genome U219 Array [12]. To better illustrate the mechanism of hepatocarcinogenesis, only HCC tissue samples with Barcelona Clinic Liver Cancer (BCLC) stage 0 were selected in this analysis. The basic information of the patients selected in this study was summarized in Table 1 (to reviewer).

\section{Identification of differentially expressed genes}

Differentially expressed genes (DEGs) between very early HCC and cirrhotic tissue samples were identified by using the Limma package on $\mathrm{R}$ language software. DEGs were considered when an adjusted $P<0.05$ and a $\mid \log _{2}$ fold change $\mid>2$. The adjusted $P$ values, by employing Benjamini and Hochberg false discovery rate, were aimed to correct the occurrence of false positive results.

Table 1 The clinical information of the patients selected

\begin{tabular}{lll}
\hline Variable & HCC & LC \\
\hline Total number & 19 & 19 \\
Median age, years & 64 & 68 \\
Gender & & 5 \\
$\quad$ Female & 6 & 14 \\
$\quad$ Male & 13 & 19 \\
Tumor stage & & 0 \\
$\quad$ Pre-cancer & 0 & \\
BLCL 0 & 19 &
\end{tabular}

HCC hepatocellular carcinoma; LC liver cirrhosis; BLCL Barcelona Clinic Liver Cancer

\section{Functional annotation of DEGs}

In this study, we used the Database for Annotation, Visualization and Integrated Discovery (DAVID) version 6.8 programs for further functional and pathways analysis of the DEGs. The DAVID analyses of the DEGs mainly contained gene ontology (GO) function analysis and Kyoto encyclopedia of genes and genomes (KEGG) pathways analysis. $\mathrm{GO}$ analysis could be further divided into three aspects, namely, biological processes (BP), cellular components (CC), and molecular functions (MF). The cutoff criterion for significant results was a $P$ value $<0.05$.

\section{Construction of PPI network and screening of hub genes}

To display the interactions and functions of the DEGs, a protein-protein interaction (PPI) network was constructed based on the database of Search Tool for the Retrieval of Interacting Genes/Proteins (STRING) in this study. All the parameters were set as defaults [13]. We applied Cytoscape 3.6.1 software to more intuitively visualize the constructed PPI network. The proteins were represented by nodes, and the protein-interactions were represented by edges in the PPI network. The molecular complex detection (MCODE) plug-in was applied to analyze the modules in the PPI networks, with the default parameters (node score $=0.2, \mathrm{~K}$-core $\geqq 2$, and $\max$ depth $=100$ ). To screen the potential hub genes that may be involved in the hepatocarcinogenesis, we applied the CytoHubba, a Cytoscape plug-in, and the maximal clique centrality algorithm.

To explore the potential clinical significance of the hub genes in HCC, we searched the Kaplan-Meier plotter, which is capable to assess the effect of 54,675 genes on survival using 18,674 cancer samples [14]. The analysis results of overall survivals (OS) with hub genes' alterations in HCC were figured with log-rank $P$ values.

\section{Results}

DEGs between early HCC and cirrhotic

A total of 200 probe IDs were identified to be differently expressed between very early $\mathrm{HCC}$ and cirrhotic tissue samples with the cutoff of adjusted $P<0.05$ and $\left|\log _{2} \mathrm{FC}\right|>2$. After matching the differed probe IDs with gene symbols from the Affymetrix dataset, 118 genes could be considered as DEGs finally.

\section{Functional enrichment analyses of DEGs}

To further understand the functions of the 118 identified DGEs, the GO and KEGG enrichment analyses of the DGEs were conducted and performed in DAVID datasets. The results of GO analysis revealed that the DGEs were mainly involved in 91 biological process terms, such as negative regulation of growth, positive regulation of B cell activation, phagocytosis and recognition, cellular response to cadmium ion, phagocytosis, and engulfment. For the 


\section{Cellular Component}

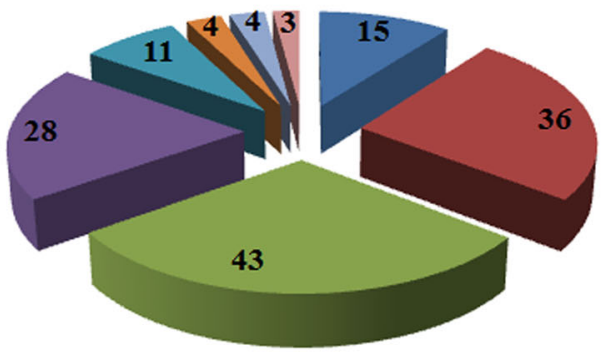

Biological Process

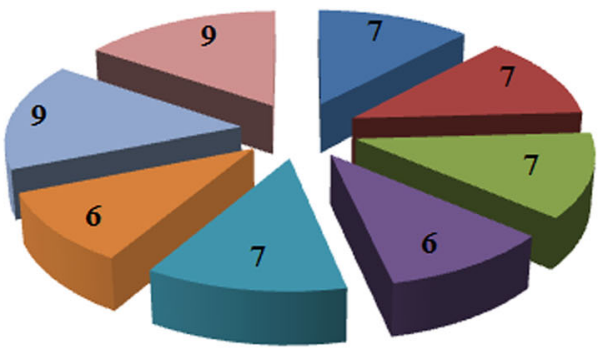

\section{Molecular Function}

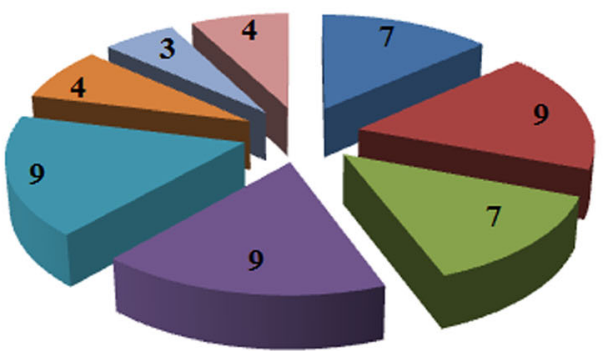

I G 0:0072562-blood microparticle

a O:0005576-ex tracellular region

nG O:0070062-ex tracellular ex osome

=G 0:0005615-ex tracellular space

=G O:0009897-ex ternal side of plasma membrane

$=\mathrm{G} \mathrm{O} 0005833$-hemoglobin complex

=G 0:0042571-imm unoglobulin complex, circulating

= G O:0031838-haptoglobin-hemoglobin complex

n G 0:0045926-negative regulation of growth

= G 0:0050871-positive regulation of $\mathrm{B}$ cell activation

= G O:0006910-phagocytosis, recognition

n G O:0071276-cellular response to cadmium ion

I G O:0006911-phagocytosis, engulfm ent

I G O:0071294-cellular response to zinc ion

W G:0006956-complem ent activation

II G O:0006958-complem ent activation, dassical pathway

n G O:0034987-imm unoglobulin receptor binding

E G O:0003823-antigen binding

a G:0019825-oxygen binding

a GO:0020037-heme binding

= G O:0005506-iron ion binding

= G O:0005344-oxygen transporter activity

M O:0031720-haptoglobin binding

= G O:0005537-mannose binding

Fig. 1 Gene ontology (GO) analysis of differentially expressed genes in very early hepatocellular carcinoma tissue samples 
Table 2 Kyoto Encyclopedia of Genes and Genomes (KEGG) pathway enrichment analyses of differentially expressed genes in very early hepatocellular carcinoma tissue samples

\begin{tabular}{llll}
\hline Term & Count & P value & Genes \\
\hline Mineral absorption & 6 & $4.71 \mathrm{E}-05$ & MT1M, MT1A, MT1H, MT1X, MT1G, MT1F \\
p53 signaling pathway & 6 & $2.88 \mathrm{E}-04$ & CDK1, CDKN2A, RRM2, IGF1, THBS1, IGFBP3 \\
African trypanosomiasis & 4 & 0.002958176 & IDO2, HBA2, HBA1, HBB \\
Tryptophan metabolism & 4 & 0.005127472 & ALDH1B1, IDO2, CYP1A2, INMT \\
Chemical carcinogenesis & 5 & 0.005272955 & CYP3A4, NAT2, HSD11B1, ADH1B, CYP1A2 \\
Malaria & 4 & 0.009039916 & HBA2, HBA1, THBS1, HBB \\
Phagosome & 6 & 0.010951526 & MRC1, MARCO, HLA-DRB4, COLEC11, THBS1, CLEC4M \\
Steroid hormone biosynthesis & 4 & 0.014316303 & CYP3A4, HSD11B1, SRD5A2, CYP1A2 \\
Metabolism of xenobiotics by cytochrome P450 & 4 & 0.027242121 & CYP3A4, HSD11B1, ADH1B, CYP1A2 \\
Salmonella infection & 4 & 0.036531167 & FOS, CCL4L1, CCL4L2, CCL4
\end{tabular}

category of cellular component, the DGEs were significantly associated with 20 terms, including blood microparticle, extracellular region, extracellular exosome, extracellular space, and external side of the plasma membrane. Moreover, for the category molecular function, the DGEs were significantly enriched with 27 terms, such as immunoglobulin receptor binding, antigen binding oxygen binding, heme binding, iron ion binding. The top eight terms in each GO category were illustrated in Fig. 1. In addition, the KEGG pathways were significantly enriched for the p53 signaling pathway, chemical carcinogenesis, as listed in Table 2.

\section{Construction of PPI network and analyses of modules}

To investigate the protein-protein interactions between the DGEs, a PPI network was constructed based on the
STRING database and was visualized by Cytoscape (Fig. 2). The PPI network consisted of 118 nodes and 203 edges. The average node degree of PPI network was 3.44 and the local clustering coefficient was 0.442 . The top two modules were chosen after analyzing the entire PPI network by employing the MCODE plug-in (Fig. 2). The hub genes were determined from the PPI network by using the cytoHubba plugin. The top eight hub genes were sequentially listed as follows: CDKN3 (cyclin-dependent kinase inhibitor 3), CDK1 (cyclin-dependent kinase 1), CCNB1 (cyclin B1), TOP2A (topoisomerase (DNA) II alpha), CCNA2 (cyclin A2), CCNB2 (cyclin B2), PRC1 (protein regulator of cytokinesis 1), and RRM2 (ribonucleotide reductase M2) (Fig. 3). All of these hub genes were found to be overexpressed in the very early HCC and included in module 1. Meanwhile, two of these hub genes, including CDK1

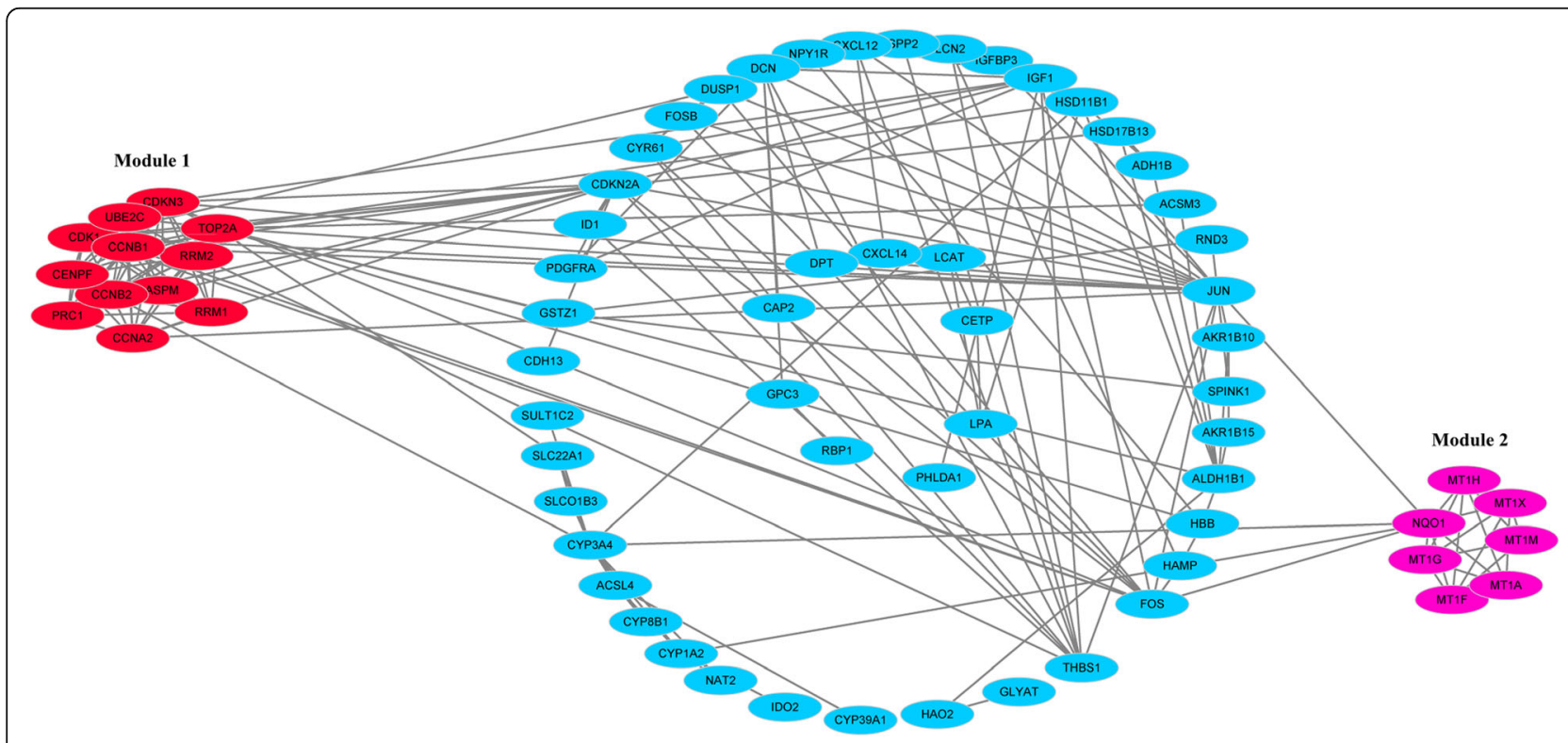

Fig. 2 Protein-protein interaction network of the differentially expressed genes in very early hepatocellular carcinoma 


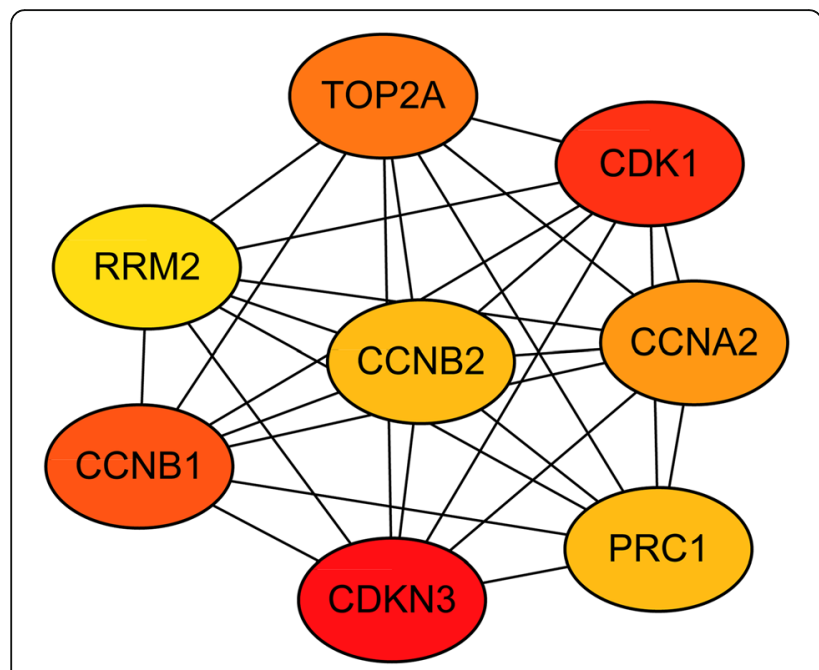

Fig. 3 Hub genes screened from protein-protein interaction network

and RRM2, were significantly enriched in the p53 signaling pathway.

\section{Exploring the prognostic value of the hub genes}

To further analyze the prognostic value of the identified hub genes, the OS associated with hub genes was analyzed for 364 patients with HCC by using the Kaplan-Meier plotter. High expressions of CDK1 $(P=1.1 \mathrm{e}-5), \mathrm{CCNB} 1(P=$ 3.4e-5), TOP2A $(P=0.0001)$, CCNA2 $(P=0.0002)$, PRC1 $(P=0.0002)$, RRM2 $(P=5.5 \mathrm{e}-5), \mathrm{CDKN} 3(P=0.0066)$, and CCNB2 $(P=0.0013)$ were associated with poor OS in HCC patients (Fig. 4).

\section{Discussion}

Although the initiation and progression of HCC had been investigated increasingly in recent years, the exact molecular mechanism of hepatocarcinogenesis is still unclear. In addition, the mortality of HCC remains high worldwide because of the limited therapeutic strategies. Thus, it is particularly urgent to dig deeper to find out the exact mechanisms underlying the development of HCC. Herein, bioinformatics analysis of gene expression profiles obtained from $\mathrm{HCC}$ may help us better to understand the molecular mechanism of tumor formation and even to find novel targets for cancer therapy. Bioinformatics analysis of HCC had been investigated in previous studies [15-17]. However, these studies were mainly focused on comparing the gene expressions between benign liver tissue samples and HCC tissue samples at early or advanced stages. Rare studies devoted to finding out the differences between very early HCC tissue samples and benign liver diseases at the gene level, which might be helpful to understand the tumor formation.
In this study, we identified a total of 118 DGEs between very early HCC and cirrhotic tissue samples chosen from the GSE63898 expression profile datasets. GO and KEGG enrichment analyses of these DGEs were also performed in DAVID datasets. The results show that the identified DGEs were closely associated with several biological processes and components, such as negative regulation of growth, extracellular region, and immunoglobulin receptor binding. Moreover, these DGEs were significantly enriched for the p53 signaling pathway, chemical carcinogenesis. Furthermore, to analyze the interactional relationships between the DGEs, a PPI network was constructed and modules analysis was done.

We had also screened out eight hub genes including CDKN3, CDK1, CCNB1, TOP2A, CCNA2, CCNB2, PRC1, and RRM2. These hub genes had been investigated extensively in the previous researches. CDKN3, also known as KAP, CDI1, CIP2, and KAP1, encodes a dual specificity protein phosphatase that had reported to dephosphorylate CDK2. CDKN3 had been overexpressed frequently in several types of cancers, such as breast cancer, prostate cancer, and HCC [18-20]. It had been reported that the overexpression of CDKN3 was correlated with the poor survival in cancer patients [18]. In this study, we found that the overexpression of CDKN3 also had significantly effect on the overall survival of HCC patients. CDK1, a member of the Ser/Thr protein kinase family, plays an essential role in the G1/S and G2/M phase transitions of eukaryotic cell cycle by interacting with CCNB1 [21]. In gastric carcinoma, high expression of CDK1 had found to lead to poor prognosis and correlated inversely with p27 expression [22]. The overexpression of CDK1 was also found to be directly associated with portal invasion, high alpha-fetoprotein level, and poor prognosis in HCC [23], which was in agreement with the results in this study.

TOP2A, encoding DNA topoisomerase II alpha, is involved in processes such as chromatid separation, cell cycle progression and the relief of torsional stress. TOP2A was reported to be frequently co-amplified with HER-2 and then reduce the clinical outcome in urinary bladder cancer and breast cancer [24, 25]. Moreover, TOP2A was also found to be significantly overexpressed and linked with poor prognosis in HCC in this study, which was in conformity with results of previous studies $[26,27]$. CCNA2 protein is functioned as a regulator of the cell cycle by activating cyclin-dependent kinases. The expression of CCNA2 was driven by E2Fs [28]. The aberrant expression of CCNA2 could be detected and closely related to reduced survival in $\mathrm{HCC}$ and breast cancer [28, 29]. CCNB2 is also a member of the cyclin family, which are core components for cell cycle control. CCNB2 was found to be overexpressed and then result 


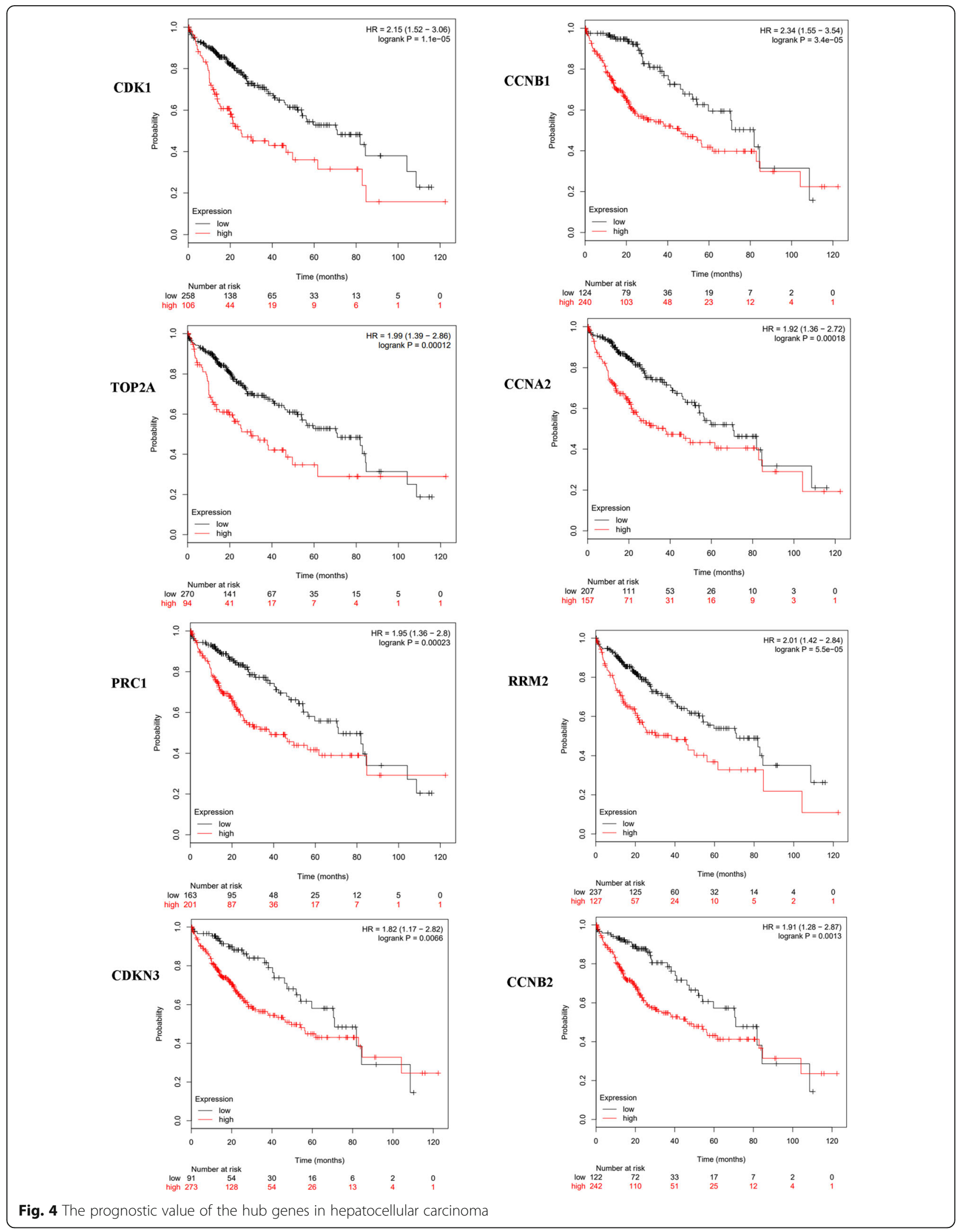


in poor prognosis in non-small-cell lung cancer and invasive breast carcinoma [30, 31].

PRC1 is necessary for microtubules organization and is a substrate of several cyclin-dependent kinases. PRC1 was reported to involve in $\mathrm{Wnt} / \beta$-catenin signaling pathway and then promote cancer proliferation and tumorigenesis in kinds of cancers, such as HCC and lung adenocarcinoma [32, 33]. RRM2 plays a critical role in converting ribonucleotides to deoxynucleotides in DNA synthesis. The elevated expression of RRM2 may lead to the poor prognosis for patients with non-small cell lung cancer by stabilization of Bcl-2, which is a critical regulator of apoptosis [34]. In addition, it had been shown RRM2 might be a novel therapeutic target in variety of cancers, such as breast cancer, non-small cell lung cancer, and bladder cancer [34-37].

\section{Conclusion}

In summary, we attempted to find out the differences between the very early HCC tissue samples and cirrhotic tissue samples at gene level by using bioinformatics analysis tools. A total of eight key genes in the initiation of $\mathrm{HCC}$ were identified and their prognostic values were explored based on Kaplan-Meier plotter. However, further experimental verification is necessary to confirm the results in this study.

\section{Abbreviations \\ CCNA2: Cyclin A2; CCNB1: Cyclin B1; CCNB2: Cyclin B2; CDK1: Cyclin- dependent kinase 1; CDKN3: Cyclin-dependent kinase inhibitor 3; DAVID: Database for Annotation, Visualization and Integrated Discovery; DEGs: Differentially expressed genes; GEO: Gene Expression Omnibus; GO: Gene ontology; HCC: Hepatocellular carcinoma; KEGG: Kyoto Encyclopedia of Genes and Genomes; OS: Overall survivals; PPI: Protein- protein interaction; PRC1: Protein regulator of cytokinesis 1: RRM2: Ribonucleotide reductase M2; STRING: Search Tool for the Retrieval of Interacting Genes/Proteins; TOP2A: Topoisomerase (DNA) II alpha}

\section{Acknowledgements}

Not applicable.

\section{Funding}

None

\section{Availability of data and materials}

The data that support the findings of this study are available from GEO database (GSE63898), DAVID, STRING, and GEPIA database, as is mentioned in the "Material and methods" section.

\section{Authors' contributions}

MW and ZL analyzed the data. WM and XL wrote the manuscript. AZ, DL, and NL supervised the study. All authors read and approved the final manuscript.

\section{Ethics approval and consent to participate}

Ethical approval was not necessary for this study because public datasets were analyzed.

\section{Consent for publication}

All authors declare that there is no conflict of interest.

\section{Competing interests}

All authors declare that they have no competing interests.

\section{Publisher's Note}

Springer Nature remains neutral with regard to jurisdictional claims in published maps and institutional affiliations.

\section{Author details}

'Department of General Surgery, Beijing Youan Hospital, Capital Medical University, 8 Xitoutiao, Youanmenwai, Feng-tai District, Beijing 100069, China. ${ }^{2}$ Beijing Institute of Hepatology, Beijing YouAn Hospital, Capital Medical University, 8 Xitoutiao, Youanmenwai, Feng-tai District, Beijing 100069, China.

Received: 19 October 2018 Accepted: 14 April 2019

Published online: 01 May 2019

\section{References}

1. Siegel RL, Miller KD, Jemal A. Cancer statistics, 2018. 2018;68(1):7-30.

2. Wu J, Yang S, Xu K, Ding C, Zhou Y, Fu X, Li Y, Deng M, Wang C, Liu X. Patterns and trends of liver cancer incidence rates in eastern and southeastern Asian countries (1983-2007) and Predictions to 2030 Gastroenterology. 2018;154(6):1719-1728.e5.

3. Ph.D HPMD, Shafritz DA, Hoofnagle JH. Relation of the hepatitis B virus carrier state to hepatocellular carcinoma. Hepatology. 2010;7(4):764-72.

4. Masahiro T, Francisco K, Hideaki K, Hideo T, Wang J, Lin QY, Manami I. Hepatitis B and C virus infection and hepatocellular carcinoma in China: a review of epidemiology and control measures. J Epidemiol. 2011; 21(6):401-16.

5. Turdean S, Gurzu S, Turcu M, Voidazan S, Sin A. Current data in clinicopathological characteristics of primary hepatic tumors. Rom J Morphol Embryol. 2012;53(3 Suppl):719.

6. Reig M, Fonseca LGD, Faivre $\mathrm{S}$. New trials and results in systemic treatment of HCC. J Hepatol. 2018;69(2):525-33.

7. Luo JJ, Zhang ZH, Liu QX, Zhang W, Wang JH, Yan ZP. Endovascular brachytherapy combined with stent placement and TACE for treatment of HCC with main portal vein tumor thrombus. Hepatol Int. 2016;10(1):185-95.

8. Cauchy F, Zalinski S, Dokmak S, Fuks D, Farges O, Castera L, Paradis V, Belghiti J. Surgical treatment of HCC associated with the metabolic syndrome. Brit J Surg. 2013;100:1.

9. Liu KY, Wang LT, Hsu SH. Modification of epigenetic histone acetylation in hepatocellular carcinoma. Cancers. 2018;10(1):8.

10. Mukhopadhyay B, Schuebel K, Mukhopadhyay P, Cinar R, Godlewski G, Xiong K, Mackie K, Lizak M, Yuan Q, Goldman D. Cannabinoid receptor promotes hepatocellular carcinoma initiation and progression through multiple mechanisms. Hepatology. 2015;61(5):1615-26.

11. Liu M, Jiang L, Guan XY. The genetic and epigenetic alterations in human hepatocellular carcinoma: a recent update. Prot Cell. 2014;5(9):673-91.

12. Villanueva A, Portela A, Sayols S, Battiston C, Hoshida Y, Méndezgonzález J, Imbeaud S, Letouzé E, Hernandezgea V, Cornella HJH. DNA methylationbased prognosis and epidrivers in hepatocellular carcinoma. Hepatology. 2015;61(6):1945-56.

13. Szklarczyk D, Morris JH, Cook H, Kuhn M, Wyder S, Simonovic M, Santos A, Doncheva NT, Roth A, Bork P, et al. The STRING database in 2017: qualitycontrolled protein-protein association networks, made broadly accessible. Nucleic Acids Res. 2017:45(D1):D362-d368.

14. Lánczky A, Nagy Á, Bottai G, Munkácsy G, Szabó A, Santarpia L, Győrffy B. miRpower: a web-tool to validate survival-associated miRNAs utilizing expression data from 2178 breast cancer patients. Breast Cancer Res Treat. 2016;160(3):439-46.

15. Zhou L, Du Y, Kong L, Zhang X, Chen Q. Identification of molecular target genes and key pathways in hepatocellular carcinoma by bioinformatics analysis. Onco Targets Ther. 2018;11:1861-9.

16. Shangguan H, Tan SY, Zhang JR. Bioinformatics analysis of gene expression profiles in hepatocellular carcinoma. Eur Rev Med Pharmacol Sci. 2015; 19(11):2054-61.

17. Li N, Li L, Chen Y. The identification of core gene expression signature in hepatocellular carcinoma. Oxid Med Cell Longev. 2018;2018(4):1-15.

18. Xing C, Xie H, Lin Z, Zhou W, Wu Z, Ding S, Wei B, Yu X, Rong S, Zheng S. Cyclin-dependent kinase inhibitor 3 is overexpressed in hepatocellular carcinoma and promotes tumor cell proliferation. Biochem Biophys Res Commun. 2012;420(1):29-35.

19. Deng M, Wang J, Chen Y, Zhang L, Xie G, Liu Q, Zhang T, Yuan P, Liu D. Silencing cyclin-dependent kinase inhibitor 3 inhibits the migration of breast cancer cell lines. Mol Med Rep. 2016;14(2):1523-30. 
20. Yu C, Cao H, He X, Sun P, Feng Y, Chen L, Gong H. Cyclin-dependent kinase inhibitor 3 (CDKN3) plays a critical role in prostate cancer via regulating cell cycle and DNA replication signaling. Biomed Pharmacother. 2017;96:1109.

21. Song YJ, Stinski MF. Inhibition of cell division by the human cytomegalovirus IE86 protein: role of the p53 pathway or cyclin-dependent kinase 1/cyclin B1. J Virology. 2005;79(4):2597-603.

22. Masuda TA, Inoue H, Nishida K, Sonoda H, Yoshikawa Y, Kakeji Y, Utsunomiya T, Mori M. Cyclin-dependent kinase 1 gene expression is associated with poor prognosis in gastric carcinoma. Clin Cancer Res. 2003; 9(15):5693-8.

23. Ito Y, Takeda T, Sakon M, Monden M, Tsujimoto M, Matsuura N. Expression and prognostic role of cyclin-dependent kinase 1 (cdc2) in hepatocellular carcinoma. Oncology. 2000;59(1):68-74.

24. Simon R, Atefy R, Wagner U, Forster T, Fijan A, Bruderer J, Wilber K, Mihatsch MJ, Gasser T, Sauter G. HER-2 and TOP2A coamplification in urinary bladder cancer. Int J Cancer. 2010;107(5):764-72.

25. Fasching $P A$, Weihbrecht $S$, Haeberle L, Gasparyan A, Villalobos IE, Ma Y, Ekici AB, Wachter DL, Hartmann A, Beckmann MW. HER2 and TOP2A amplification in a hospital-based cohort of breast cancer patients: associations with patient and tumor characteristics. Breast Cancer Res Treat. 2014;145(1):193-203.

26. Panvichian R, Tantiwetrueangdet A, Angkathunyakul N, Leelaudomlipi S. TOP2A amplification and overexpression in hepatocellular carcinoma tissues. Biomed Res Int. 2015;2015:381602.

27. Han Z, Zhang M, Zhang Y, Wang Y, Du X, Oncology DO. Overexpression of TOP2A in non- small cell lung cancer promotes cancer cell proliferation and invasion. J Modern Oncol. 2016

28. Gopinathan L, Tan SL, Padmakumar VC, Coppola V, Tessarollo L, Kaldis P. Loss of Cdk2 and cyclin A2 impairs cell proliferation and tumorigenesis. Cancer Res. 2014;74(14):3870-9.

29. Bukholm IR, Bukholm G, Nesland JM. Over-expression of cyclin A is highly associated with early relapse and reduced survival in patients with primary breast carcinomas. Int J Cancer. 2010;93(2):283-7.

30. Qian X, Song X, He Y, Yang Z, Sun T, Wang J, Zhu G, Xing W, You C. CCNB2 overexpression is a poor prognostic biomarker in Chinese NSCLC patients. Biomed Pharmacother. 2015;74:222-7.

31. Shubbar E, Kovács A, Hajizadeh S, Parris TZ, Nemes S, Gunnarsdóttir K, Einbeigi Z, Karlsson P, Helou K. Elevated cyclin B2 expression in invasive breast carcinoma is associated with unfavorable clinical outcome. BMC Cancer. 2013;13(1):1-10.

32. Chen J, Rajasekaran M, Xia H, Zhang X, Kong SN, Sekar K, Seshachalam VP, Deivasigamani A, Goh BKP, Ooi LL. The microtubule-associated protein PRC1 promotes early recurrence of hepatocellular carcinoma in association with the Wnt/ $\beta$-catenin signalling pathway. Gut. 2016;65(9):1522-34.

33. Zhan P, Zhang B, Xi GM, Wu Y, Liu HB, Liu YF, Xu WJ, Zhu QQ, Cai F, Zhou ZJ. PRC1 contributes to tumorigenesis of lung adenocarcinoma in association with the Wnt/B-catenin signaling pathway. Mol Cancer. 2017; 16(1):108.

34. Rahman MA, Amin AR, Wang D, Koenig L, Nannapaneni S, Chen Z, Wang Z, Sica G, Deng X, Chen ZG. RRM2 regulates BCl-2 in head and neck and lung cancers: a potential target for cancer therapy. Clin Cancer Res. 2013;19(13): 3416-28.

35. Rasmussen RD, Gajjar MK, Tuckova L, Jensen KE, Maya-Mendoza A, Holst CB, Møllgaard K, Rasmussen JS, Brennum J, Bartek J Jr. BRCA1-regulated RRM2 expression protects glioblastoma cells from endogenous replication stress and promotes tumorigenicity. Nat Commun. 2016;7:13398.

36. Afrasiabi A, Fiuji F, Mirhafez R, Avan A. RRM2 (ribonucleotide reductase M2). 2015;1.

37. Morikawa T, Maeda D, Kume H, Homma Y, Fukayama M. Ribonucleotide reductase $M 2$ subunit is a novel diagnostic marker and a potential therapeutic target in bladder cancer. Histopathology. 2011:57(6):885-92.

Ready to submit your research? Choose BMC and benefit from:

- fast, convenient online submission

- thorough peer review by experienced researchers in your field

- rapid publication on acceptance

- support for research data, including large and complex data types

- gold Open Access which fosters wider collaboration and increased citations

- maximum visibility for your research: over $100 \mathrm{M}$ website views per year

At $\mathrm{BMC}$, research is always in progress.

Learn more biomedcentral.com/submissions 